\title{
Evaluation of interpolation methods for TG-43 dosimetric parameters based on comparison with Monte Carlo data for high-energy brachytherapy
}

\section{sources}

\author{
Ma Carmen Pujades-Claumarchirant, MSc'.o), Domingo Granero, PhD², Jose Perez-Calatayud, PhD', \\ Facundo Ballester, $\mathrm{PhD}^{3}$, Christopher Melhus, $\mathrm{PhD}^{4}$, Mark Rivard, $\mathrm{PhD}^{4}$ \\ IPhysics Section, Department of Radiation Oncology, La Fe University Hospital, Valencia, Spain, 2Department of Radiation Oncology, ERESA. \\ Hospital General Universitario, Valencia, Spain, ${ }^{3}$ Department of Atomic, Molecular and Nuclear Physics, University of Valencia, Burjassot, \\ Valencia, Spain, ${ }^{4}$ Department of Radiation Oncology, Tufts University School of Medicine, Boston, Massachusetts, USA
}

\begin{abstract}
Purpose: The aim of this work was to determine dose distributions for high-energy brachytherapy sources at spatial locations not included in the radial dose function $g_{\mathrm{L}}(r)$ and $2 \mathrm{D}$ anisotropy function $F(r, \theta)$ table entries for radial distance $r$ and polar angle $\theta$. The objectives of this study are as follows: 1) to evaluate interpolation methods in order to accurately derive $g_{\mathrm{L}}(r)$ and $F(r, \theta)$ from the reported data; 2$)$ to determine the minimum number of entries in $g_{\mathrm{L}}(r)$ and $F(r, \theta)$ that allow reproduction of dose distributions with sufficient accuracy.

Material and methods: Four high-energy photon-emitting brachytherapy sources were studied: ${ }^{60} \mathrm{Co}$ model Co0.A86, ${ }^{137} \mathrm{Cs}$ model CSM-3, ${ }^{192}$ Ir model Ir2.A85-2, and ${ }^{169} \mathrm{Yb}$ hypothetical model. The mesh used for $r$ was: $0.25,0.5,0.75,1,1.5$, 2-8 (integer steps) and $10 \mathrm{~cm}$. Four different angular steps were evaluated for $F(r, \theta): 1^{\circ}, 2^{\circ}, 5^{\circ}$ and $10^{\circ}$. Linear-linear and logarithmic-linear interpolation was evaluated for $g_{\mathrm{L}}(r)$. Linear-linear interpolation was used to obtain $F(r, \theta)$ with resolution of $0.05 \mathrm{~cm}$ and $1^{\circ}$. Results were compared with values obtained from the Monte Carlo (MC) calculations for the four sources with the same grid.

Results: Linear interpolation of $g_{\mathrm{L}}(r)$ provided differences $\leq 0.5 \%$ compared to MC for all four sources. Bilinear interpolation of $F(r, \theta)$ using $1^{\circ}$ and $2^{\circ}$ angular steps resulted in agreement $\leq 0.5 \%$ with $\mathrm{MC}$ for ${ }^{60} \mathrm{Co}$, ${ }^{192} \mathrm{Ir}$, and ${ }^{169} \mathrm{Yb}$, while ${ }^{137} \mathrm{Cs}$ agreement was $\leq 1.5 \%$ for $\theta<15^{\circ}$.

Conclusions: The radial mesh studied was adequate for interpolating $g_{\mathrm{L}}(r)$ for high-energy brachytherapy sources, and was similar to commonly found examples in the published literature. For $F(r, \theta)$ close to the source longitudinalaxis, polar angle step sizes of $1^{\circ}-2^{\circ}$ were sufficient to provide $2 \%$ accuracy for all sources.
\end{abstract}

J Contemp Brachyther 2010; 2, 1: 28-32

Key words: brachytherapy, dosimetry, TG-43, interpolation, radial dose function, 2D anisotropy function.

\section{a) This work was presented in part at the ABS annual meeting in Toronto (Canada), 2009 and in ESTRO annual meeting in Göteborg (Sweden), 2008}

\section{Purpose}

Treatment planning systems (TPS) used in brachytherapy, employ the American Association of Physicists in Medicine (AAPM) Task Group No. 43 Report (TG-43) formalism $[1,2]$ in which the radial dose function $g_{\mathrm{L}}(r)$ and 2D anisotropy function $F(r, \theta)$ are introduced in the form of single and double entry tables, respectively, using a spe- cific mesh for each parameter. Current TPS require dose calculation in a clinical implant using higher spatial resolution of radial distance $\mathrm{r}$ and polar angle $\theta$ than the entered parameter data, i.e., $g_{\mathrm{L}}(r)$ and $F(r, \theta)$. Therefore, TPS must interpolate $g_{\mathrm{L}}(r)$ and $F(r, \theta)$ values from data tables.

A review of the published data for various brachytherapy sources indicated that different authors used a variety
Address for correspondence: M. Carmen Pujades Claumarchirant, MSc, Physics Section, Department of Radiation Oncology, La Fe University Hospital, Avda. Campanar 21, 46009, Valencia, Spain, phone +34963862700, ext. 50536, fax +34963862700, e-mail: mpuclau@gmail.com
Received: 15.02 .10

Accepted: 02.03 .10

Published: 28.03.10 
of spatial and angular increments and ranges in their reporting. Therefore, a standardized methodology for interpolation from the published data may be required to determine the dose rate distributions at spatial locations not explicitly included in the published data. The AAPM TG-43U1 [2] report provided guidelines for interpolation from $2 \mathrm{D}$ and 1D dosimetry parameters for the case of low energy sources of ${ }^{125} \mathrm{I}$ and ${ }^{103} \mathrm{Pd}$. The supplement to $2004 \mathrm{TG}-43$ report (i.e., TG-43U1S1) [3] included further clarification and modifications of the interpolation techniques in order to assemble these procedures as more accurate and user-friendly.

The TG-43U1 and TG-43U1S1 reports recommended log-linear interpolation for $g_{\mathrm{L}}(r)$ and linear-linear interpolation for $F(r, \theta)$. An accuracy of $\pm 2 \%$ was required for establishing $r$ and $\theta$ resolution, interpolation techniques and fitting procedures. The TG-43U1S1 indicated that these interpolation techniques may be extended to other brachytherapy sources in general. Polynomial fits are usually included, although tri-exponential fits and other fitting functions recently have been explored with very good agreement for all sources [4-9].

The TG-43 formalism has also been extended for highenergy sources of ${ }^{60} \mathrm{Co},{ }^{137} \mathrm{Cs}$, ${ }^{192} \mathrm{Ir}$ and ${ }^{169} \mathrm{Yb}$ [10]. However, given the contradictory behaviour of $g_{\mathrm{L}}(r)$ and $F(r, \theta)$ between low-energy and high-energy brachytherapy sources due to photon interactions, it is quite interesting to determine whether the TG-43U1 and TG-43U1S1 recommendations on interpolation and extrapolation for low-energy sources are applicable to high-energy sources [11, 12]. Therefore, the objectives of this study are: 1) to check what interpolation method allows accurate acquisition of $g_{\mathrm{L}}(r)$ and $F(r, \theta)$ from the published data; 2$)$ to determine the minimum number of entries in $g_{\mathrm{L}}(r)$ and $F(r, \theta)$ that allow reproduction of dose distributions with sufficient accuracy.

\section{Material and methods}

Four high-energy photon-emitting brachytherapy sources were studied in the present work: (1) ${ }^{60}$ Co source from BEBIG (model Co0.A86) [13]; (2) ${ }^{137}$ Cs source from BEBIG (model CSM-3) [14]; (3) 192Ir source from BEBIG (model Ir2.A85-2) [15]; and (4) a hypothetical ${ }^{169} \mathrm{Yb}$ source having the same design as ${ }^{192}$ Ir Flexisource from Isodose Control [16], but with central core composed of ${ }^{169} \mathrm{Yb}$. These sources represents the typical high-energy sources in shape and material composition. All four sources had active lengths $L=0.35 \mathrm{~cm}$ with the exception of ${ }^{137} \mathrm{Cs}$ source that had an equivalent active length (number of seeds times separation between sources) of $L=1.8 \mathrm{~cm}$ [17].

For these sources, we used the Monte Carlo (MC) raw data, $\dot{D}(r, \theta)$, in a mesh of $0.5 \mathrm{~mm}$ from 0 to $10 \mathrm{~cm}$ in $\theta=1^{\circ}$ steps obtained in previous publications [13-15], and performed equivalent simulations for ${ }^{169} \mathrm{Yb}$ theoretical source. The $g_{\mathrm{L}}(r)$ and $F(r, \theta)$ brachytherapy dosimetry parameters were derived using this dense mesh. Detailed description of the MC study of ${ }^{60} \mathrm{Co},{ }^{137} \mathrm{Cs}$ and ${ }^{192} \mathrm{Ir}$ sources can be found in respective publications. The study for ${ }^{169} \mathrm{Yb}$ source has been performed with the same methodology as for the other. A summary of methodology employed is presented below:
(1) Geant4 toolkit was used [18].

(2) Cross-section libraries based on EPDL97 [19].

(3) Radiation spectra was adopted from the National Nuclear Data Center (NNDC) [20].

(4) Water- and air-kerma per photon history were scored using linear track-length estimator of energy deposition.

(5) Each source was placed at the centre of a spherical water phantom with radius $R=40 \mathrm{~cm}$, except for ${ }^{60} \mathrm{Co}$ where the radius used was $50 \mathrm{~cm}$. Kerma estimation in water used spherical voxels that were arranged every $0.05 \mathrm{~cm}$ in $1^{\circ}$ steps.

(6) Source materials considered were assumed from the corresponding publication of each source.

(7) Water and air composition and conditions were recommended by the TG-43U1.

(8) Photons were generated uniformly and distributed within the active source core.

(9) The quantity of simulated photon histories was sufficient enough to assure good statistical uncertainties (see each publication for additional details).

Published $g_{\mathrm{L}}(r)$ and $F(r, \theta)$ tables for high-energy sources used a radial mesh for $r$ that typically includes a combination of $0.25,0.5,0.75,1,1.5,2-8$ (integer steps) and $10 \mathrm{~cm}$. Some authors may add supplementary data points for $r<10 \mathrm{~cm}[21,22]$ or at larger distances such as $r=12 \mathrm{~cm}$ or $r=15 \mathrm{~cm}$ [13-16]. In case of $F(r, \theta)$, the typical spatial resolution for $\theta$ is $0^{\circ}-5^{\circ}$ (in $1^{\circ}$ steps), $5^{\circ}-10^{\circ}$ (in $2^{\circ}$ steps), $10^{\circ}-30^{\circ}$ (in $5^{\circ}$ steps), $30^{\circ}-90^{\circ}$ (in $10^{\circ}$ steps) with the same possibility of supplementary angles. In some studies of high-energy sources, lower angular resolutions were used such as $10^{\circ}$ near the source longitudinal-axis $[21,22]$. For the four sources examined, the published $g_{\mathrm{L}}(r)$ and $F(r, \theta)$ tables used the typical mesh as previously indicated. The TG-43U1 and TG-43U1S1 interpolation recommendations were examined in this context. Furthermore, linear-linear interpolation of $g_{\mathrm{L}}(r)$ was examined. Results were compared with the values obtained from the MC calculations for the aforementioned sources with the same grid.

\section{Results}

Results for linear-linear interpolation and logarithmiclinear interpolation did not differ significantly for $g_{\mathrm{L}}(r)$ as shown in Fig. 1. For ${ }^{192} \mathrm{Ir}$ and ${ }^{162} \mathrm{Yb}$ sources, interpolation differences were $\leq 0.5 \%$ compared with $\mathrm{MC}$ results over the entire radial range $0.25 \leq r \leq 10 \mathrm{~cm}$. For ${ }^{60} \mathrm{Co}$ and ${ }^{137} \mathrm{Cs}$ sources, differences between $\mathrm{MC}$ and interpolation results were $>2 \%$ for $0.25<r<0.5 \mathrm{~cm}$ and $\leq 0.5 \%$ elsewhere. Dissimilarities between $\mathrm{MC}$ and interpolation results reduced to $\leq 0.5 \%$ upon addition of $g_{\mathrm{L}}(r=0.33 \mathrm{~cm})$ and $g_{\mathrm{L}}(r=0.35$ $\mathrm{cm})$ for ${ }^{60} \mathrm{Co}$ and ${ }^{137} \mathrm{Cs}$, respectively, to account for $g_{\mathrm{L}}(r)$ maximum in the case of ${ }^{60} \mathrm{Co}$, and the high $g_{\mathrm{L}}(r)$ gradient in the case of ${ }^{137} \mathrm{Cs}$ (Fig. 2). Due to small $(<0.5 \%)$ rounding errors in the published data, the $g_{\mathrm{L}, \text { Int. }}(r) / g_{\mathrm{L}, \mathrm{MC}}(r)$ ratio is not equal to unity at radii corresponding to the tabulated data points. There were no substantial differences between the linear-linear and log-linear interpolations for the four sources examined. 
A

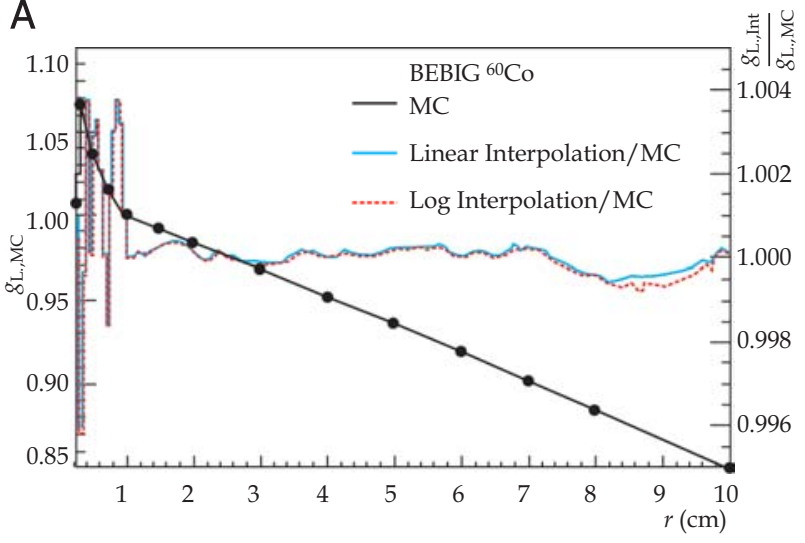

C

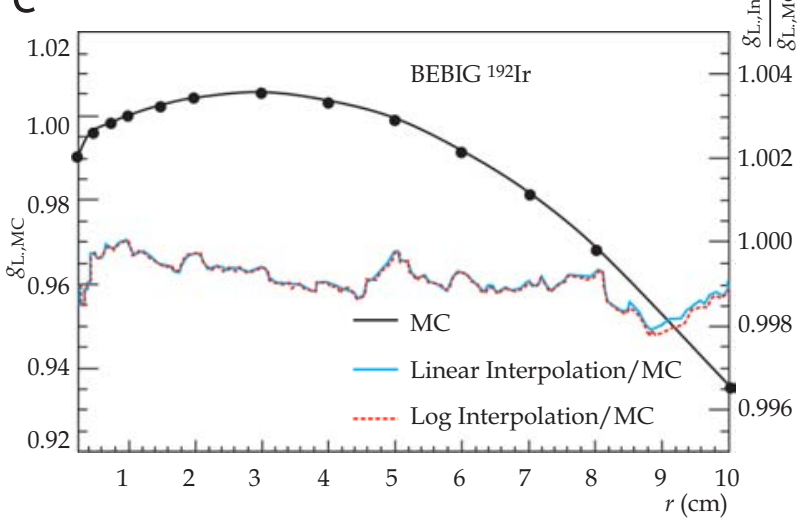

B

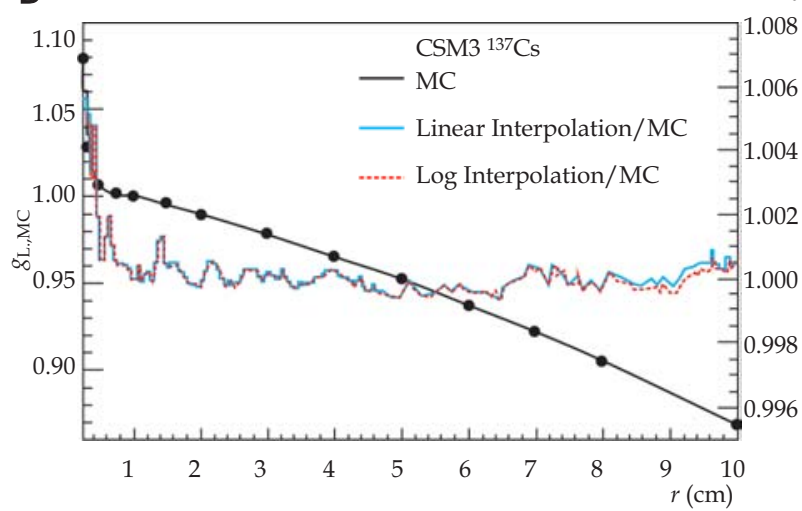

D

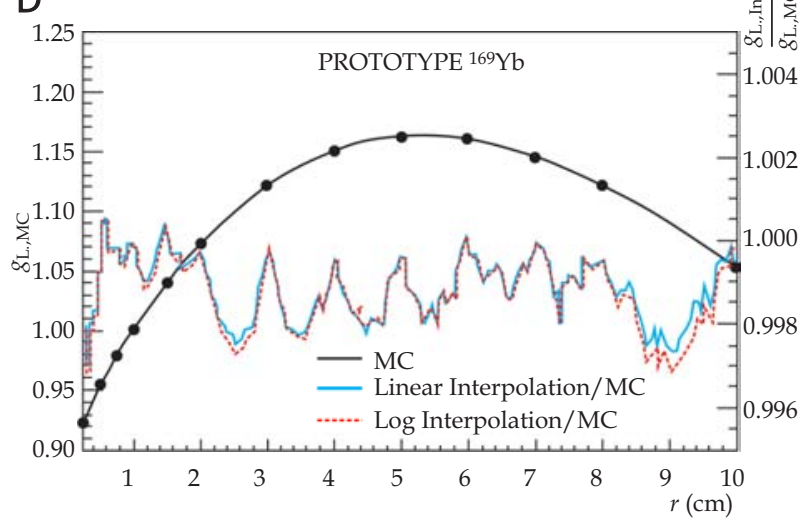

Fig. 1. Radial dose function $g_{L}(r)$ for the four sources studied (left scale) and ratio of interpolated $g_{L}(r)$ to MC raw data (right scale). Full black lines represent MC results in a mesh of $0.5 \mathrm{~mm}$ from 0 to $10 \mathrm{~cm}$. Closed circles represent the same MC results but for the radial mesh typically used in published tables. The mesh points used for interpolation are shown as closed circles

The results for $F(r, \theta)$ are shown in Table 1, with graphical representation for ${ }^{192}$ Ir source shown in Fig. 3. For the four sources and four approximations in case of $F(r, \theta)$, differences with $\mathrm{MC}$ were $\leq 0.5 \%$ in the radial range up to $10 \mathrm{~cm}$ when using $1^{\circ}$ and $2^{\circ}$ polar angle steps, with the exception of ${ }^{137} \mathrm{Cs}$ where differences were $\leq 1.5 \%$ for $\theta<15^{\circ}$. With $5^{\circ}$ polar angle steps, differences for ${ }^{60} \mathrm{Co},{ }^{137} \mathrm{Cs}$, ${ }^{192} \mathrm{Ir}$, and ${ }^{169} \mathrm{Yb}$ sources were $\leq 0.5 \%, \leq 1.5 \%$ for $\theta<15^{\circ}, \leq 0.5 \%$, and $\leq 2 \%$ for $\theta<5^{\circ}$, respectively. With $10^{\circ}$ polar angle steps, dissimilarities for ${ }^{60} \mathrm{Co},{ }^{137} \mathrm{Cs},{ }^{192} \mathrm{Ir}$, and ${ }^{169} \mathrm{Yb}$ sources were $\leq 1.5 \%$ for $\theta<5^{\circ}, \leq 2 \%$ for $\theta<10^{\circ}, \leq 1.5 \%$ for $\theta<25^{\circ}$, $\leq 2 \%$ for $\theta<10^{\circ}$.

\section{Discussion}

If dosimetric information is required (i.e., desire to evaluate organ-at-risk dose) for $r>10 \mathrm{~cm}$, physicists should refer to the original MC publications. However, radiation scatter conditions and the water equivalence of tissues may need to be considered for accurate dose estimation [23].

In contrast with dosimetry parameter interpolation for low-energy brachytherapy sources, extrapolation to $r \leq r_{\text {rmin }}$ for high-energy sources is complicated by the lack of electronic equilibrium and the assumption that collisional kerma is equal to absorbed dose over the entire radial range. Significant issues that are generally not included in most publications on high-energy brachytherapy source dosimetry are the presence of electronic disequilibrium near the source and the contributions from emitted electrons [24]. Consequently, no extrapolation method can predict the behaviour of data without obtaining the physical basis in order to understanding the effect.

\section{Conclusions}

In contrast to the established standards (TG-43U1S1) for low-energy sources which recommends log-linear interpolation for $g_{\mathrm{L}}(r)$, linear-linear or log-linear interpolation methods, produced nearly the same results for high-energy sources. For $g_{\mathrm{L}}(r)$ and for sources analysed in this study, the typical mesh used in the literature was adequate for linear-linear or log-linear interpolations of ${ }^{192} \mathrm{Ir}$ and ${ }^{169} \mathrm{Yb}$ sources. For ${ }^{60} \mathrm{Co}$ and ${ }^{137} \mathrm{Cs}$, the mesh was also adequate for $g_{\mathrm{L}}(r)$, however an additional $g_{\mathrm{L}}(r)$ point for $0.25<r<$ $0.5 \mathrm{~cm}$ was included to keep minimize interpolation errors to $<0.5 \%$. For $F(r, \theta)$ close to longitudinal axis source (i.e., $\left.\theta<15^{\circ}\right), 1^{\circ}-2^{\circ}$ polar angle steps were adequate for all 4 sources examined.

\section{Acknowledgments}

This study was supported in part by the Generalitat Valenciana (Project GVEMP06/015), Ministerio de Educación y Ciencia (Spain) Projects No. FIS2004-05713, 

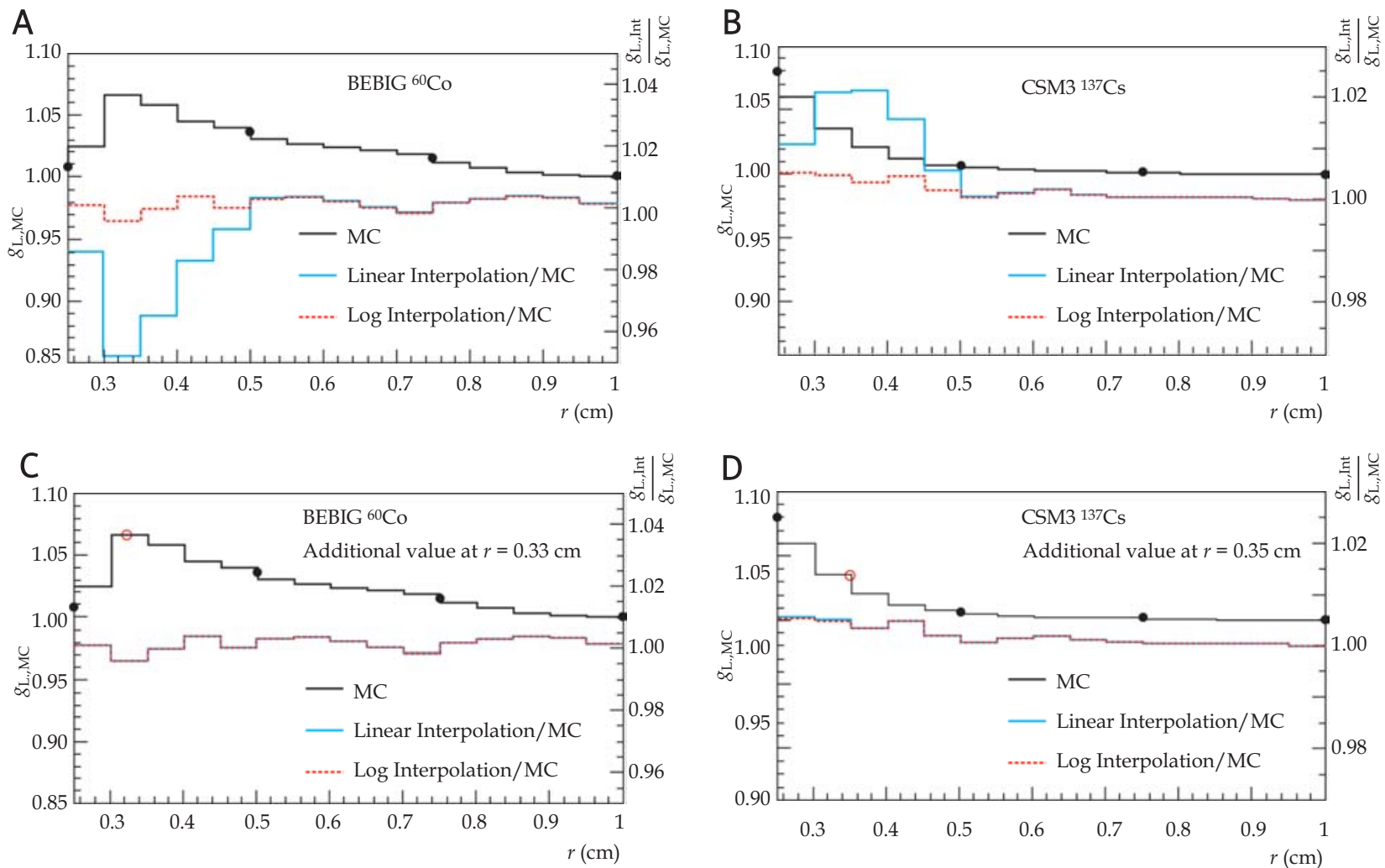

Fig. 2. Radial dose fuction $g_{L}(r)$ for the two sources studied in the radial range up to $1 \mathrm{~cm}$ (left) and with two additional points (right)

Table 1. Differences of linear-linear interpolated $F(r, \theta)$ values compared to $M C$ results

\begin{tabular}{ccccc}
$\theta$ step & ${ }^{60} \mathrm{Co}$ & ${ }^{137} \mathrm{Cs}$ & ${ }^{192} \mathrm{rr}$ & \\
\hline $1^{\circ}-2^{\circ}$ & $\leq 0.5 \%$ & $\leq 1.5 \%\left(\theta<15^{\circ}\right)$ & $\leq 0.5 \%$ \\
& & $\leq 0.5 \%\left(\theta>15^{\circ}\right)$ & \\
\hline $5^{\circ}$ & $\leq 0.5 \%$ & $\leq 1.5 \%\left(\theta<15^{\circ}\right)$ & $\leq 0.5 \%$ & $\leq 2 \%\left(\theta<5^{\circ}\right)$ \\
& & $\leq 0.5 \%\left(\theta>15^{\circ}\right)$ & & $\leq 0.5 \%\left(\theta>5^{\circ}\right)$ \\
\hline $10^{\circ}$ & $\leq 1.5 \%\left(\theta<5^{\circ}\right)$ & $\leq 2 \%\left(\theta<10^{\circ}\right)$ & $\leq 1.5 \%\left(\theta<25^{\circ}\right)$ & $\leq 2 \%\left(\theta<10^{\circ}\right)$ \\
& $\leq 0.5 \%\left(\theta>5^{\circ}\right)$ & $\leq 0.5 \%\left(\theta>10^{\circ}\right)$ & $\leq 0.5 \%\left(\theta>25^{\circ}\right)$ & $\leq 0.5 \%\left(\theta>10^{\circ}\right)$
\end{tabular}
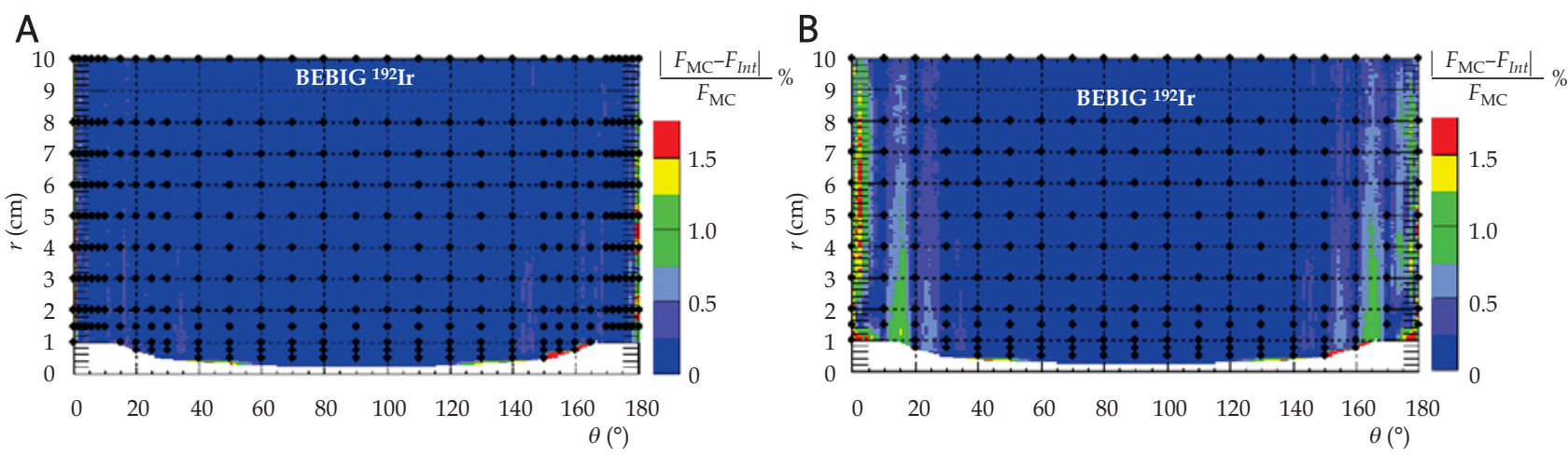

Fig. 3. Comparison between interpolated $\left(F_{i n t}\right)$ and MC $\left(F_{M C}\right) 2 \mathrm{D}$ anisotropy function results for the BEBIG ${ }^{192}$ Ir source for two different angular resolutions: a) $2^{\circ}$ increments and b) $10^{\circ}$ increments. The mesh points (closed circles) used for interpolation are shown. The region inside the source capsule is shown in white near $r=0$ and is not clinically relevant 
DPI2004-04268-C02-01 and FPA2003-07581-C02-01, and by FEDER. M.C. Pujades-Claumarchirant was supported by Fundación FIVO (Valencia, Spain).

\section{References}

1. Nath R, Anderson LL, Luxton G et al. Dosimetry of interstitial brachytherapy sources: Recommendations of the AAPM Radiation Therapy Committee Task Group No. 43. Med Phys 1995; 22: 209-234.

2. Rivard MJ, Coursey BM, DeWerd LA et al. Update of AAPM Task Group No. 43 Report: A revised AAPM protocol for brachytherapy dose calculation. Med Phys 2004; 31: 633-674.

3. Rivard MJ, Butler WM, DeWerd LA et al. Supplement to the 2004 update of the AAPM Task Group No. 43 Report. Med Phys 2007; 34: 2187-2205.

4. Wu X, Brezovich IA, Fiveash JB. Bi- and tri-exponential fitting to TG-43 radial dose functions of brachytherapy sources based on a genetic algorithm. Brachytherapy 2009; 8: 361-366.

5. Taylor RPE, Rogers DWO. More accurate fitting of ${ }^{125} \mathrm{I}$ and 103Pd radial dose functions. Med Phys 2008; 35: 4242-4250.

6. Furhang EE and Wallace RE. Fitting and benchmarking of dosimetry data for new brachytherapy sources. Med Phys 2000; 27: 2302-2306.

7. Fung AY. Comment on "Functional fitting of interstitial brachytherapy dosimetry data recommended by the AAPM Radiation Therapy Committee Task Group 43"' Med Phys 1999; 26: 153-160] and "Fitting and benchmarking of dosimetry data for new brachytherapy sources"'[Med Phys 2000; 27: 23022306]. Med Phys 2007; 28: 400.

8. Lliso F, Perez-Calatayud J, Carmona V et al. Fitted dosimetric parameters of high dose-rate ${ }^{192}$ Ir sources according to the AAPM TG43 formalism. Med Phys 2001; 28: 654-660.

9. Lliso F, Perez-Calatayud J, Carmona V et al. Technical note: Fitted dosimetric parameters of high dose-rate 192Ir sources according to the AAPM TG43 formalism. Med Phys 2003; 30: 651654.

10. Li Z, Das R K, DeWerd L A et al. Dosimetric prerequisites for routine clinical use of photon emitting brachytherapy sources with average energy higher than $50 \mathrm{keV}$. Med Phys 2007; 34: 37-40.

11. Sakelliou L, Sakellariou K, Sarigiannis K et al. Dose rate distributions around ${ }^{60} \mathrm{Co},{ }^{137} \mathrm{Cs},{ }^{198} \mathrm{Au},{ }^{192} \mathrm{Ir},{ }^{241} \mathrm{Am},{ }^{125} \mathrm{I}$ (models 6702 and 6711) brachytherapy sources and the nuclide ${ }^{99} \mathrm{Tcm}$. Phys Med Biol 1992; 37: 1859-1872.

12. Mainegra E, Capote R, López E. Radial dose functions for ${ }^{103} \mathrm{Pd},{ }^{125} \mathrm{I},{ }^{192} \mathrm{Ir}$ and ${ }^{169} \mathrm{Yb}$ brachytherapy sources: an EGS4 Monte Carlo study. Phys Med Biol 2000; 45: 703-717.

13. Granero D, Perez-Calatayud J, Ballester F. Technical note: Dosimetric study of a new Co-60 source used in brachytherapy. Med Phys 2007; 34: 3485-3488.

14. Perez-Calatayud J, Granero D, Casal E et al. Monte Carlo and experimental derivation of TG-43 dosimetric parameters for CSM-type Cs-137 sources. Med Phys 2005: 28-36.

15. Granero D, Perez-Calatayud J, Ballester F. Monte Carlo study of the dose rate distributions for the Ir2.A85-2 and Ir2.A85-1 Ir-192 afterloading sources. Med Phys 2008; 35: 1280-1287.

16. Granero D, Perez-Calatayud J, Casal E et al. A dosimetric study on the Ir-192 HDR Flexisource. Med Phys 2006; 33: 4578-4582.

17. Williamson JF. Monte Carlo and analytic calculation of absorbed dose near ${ }^{137} \mathrm{Cs}$ intracavitary sources. Int J Radiat Oncol Biol Phys 1988; 15: 227-237.

18. Agostinelli S, Allison J, Amako K et al. GEANT4 - a Simulation Toolkit. Nuc Ins Meth 2003; A506: 250-303. See also http://geant4.web.cern.ch/geant4 last accessed 14 February 2010.

19. Cullen DE, Hubbell JH, Kissel L. EPDL97: The Evaluated Photon Data Library, '97 Version. Lawrence Livermore National Laboratory report UCRL-50400 1997; 6 Revision 5.
20. NUDAT 2.5, National Nuclear Data Center, Brookhaven National Laboratory, http:/ / www.nndc.bnl.gov/nudat2/ last accessed 14 February 2010.

21. Medich DC, Munro JJ. Monte Carlo characterization of the M19 high dose rate Iridium-192 brachytherapy source. Med Phys 2007; 34: 1999-2006.

22. Medich DC, Tries MA, Munro JJ. Monte Carlo characterization of an Ytterbium-169 high dose rate brachytherapy source with analysis of statical uncertainty. Med Phys 2006; 33: 163-172.

23. Rivard MJ, Venselaar JLM, Beaulieu L. The evolution of brachytherapy treatment planning systems. Med Phys 2009; 36: 2136-2153.

24. Ballester F, Granero D, Perez-Calatayud J et al. Evaluation of high-energy brachytherapy source electronic disequilibrium and dose from emitted electrons. Med Phys 2009; 36: 4250-4256. 